\title{
Stochastic dynamics of the cardiovascular system
}

\author{
Peter V.E. McClintock ${ }^{a}$ and Aneta Stefanovska ${ }^{b, a}$ \\ ${ }^{a}$ Department of Physics, Lancaster University, Lancaster LA1 4YB, UK \\ ${ }^{b}$ Nonlinear Dynamics and Synergetics, Faculty of Electrical Engineering, \\ University of Ljubljana, Tržaška 25, 1000 Ljubljana, Slovenia
}

\begin{abstract}
The human cardiovascular system (CVS), responsible for the delivery of nutrients and removal of waste products to/from the entire body, is a highly complex system involving many control mechanisms. Signals derived from the CVS are inherently difficult to analyse because they are noisy, time-varying, and of necessarily limited duration. The application of techniques drawn from nonlinear science has, however, yielded many insights into the nature of the CVS, and has provided strong evidence for a large degree of determinism in the way it functions. Yet there is compelling evidence that random fluctuations (noise) also play an essential role. There are at least five oscillatory processes of widely differing frequency involved in the blood distribution. The evidence for them, and their probable physiological origins, are discussed. Interactions between some of the processes can give rise to modulation and synchronization phenomena, very similar to those observed in classical oscillators in many areas of physics. The extent to which the CVS can be modelled as a stochastic nonlinear dynamical system is reviewed, and future research directions and possible applications based on this perception are considered.
\end{abstract}

Keywords: cardiovascular system, noise, coupled oscillators, inference, stochastic nonlinear models

\section{INTRODUCTION}

\subsection{The cardiovascular system}

The cardiovascular system (CVS) consists of the heart, lungs and blood vessels. Its function is to keep the blood in continuous circulation, carrying nutrients and oxygen to all parts of the body and removing metabolic waste products. Starting from the left heart, the blood passes through the aorta, arteries, and capillaries. It is in the capillary bed that most of the exchange of nutrients takes place, including the take up of oxygen by the cells and the corresponding removal of carbon dioxide. The blood flows from the capillaries into venules, and then passes via the veins and vena cava to the right heart, whence it is pumped via the pulmonary artery to the lungs. After giving up carbon dioxide there and becoming re-oxygenated, the blood returns via the pulmonary vein to the left heart, and the process repeats. In a resting human, the whole volume of blood $(\sim 5 \ell)$ flows through the heart within about one minute, on average.

To ensure effective function of the CVS, while keeping the blood pressure within appropriate limits, close coordination between its components is clearly essential. Correspondingly, there are several control mechanisms that are able to adjust the heart rate and stroke volume, and the impedance of the vascular system. The blood vessels that comprise the latter are very far from being inert tubes. Rather, they have muscular walls that exhibit vasomotion - continuous oscillatory movements that are used to regulate the flow.

In what follows we consider the CVS as a single entity made up of the relatively small number of subsystems discussed above. We will not be interested in the detail of how e.g. the heart functions, or in its internal structure. Instead, we consider it as a discrete subsystem, able to interact in complex ways with the other subsystems that constitute the CVS. We will show that many features of the cardiovascular system can be accounted for if it is treated as a stochastic dynamical system using techniques drawn from nonlinear science.

We emphasize that our review amounts to a status report on work in progress, and that there are many interesting questions raised by the research that still remain to be answered. Although the approach that we will describe seems highly promising in relation to applications, these are still in their infancy as we discuss below. It is our hope that this review will be useful to those who wish to develop the techniques in question, especially in relation to their many potential applications in the medical field.

E-mails: p.v.e.mcclintock@lancaster.ac.uk and aneta@osc.fe.uni-lj.si 

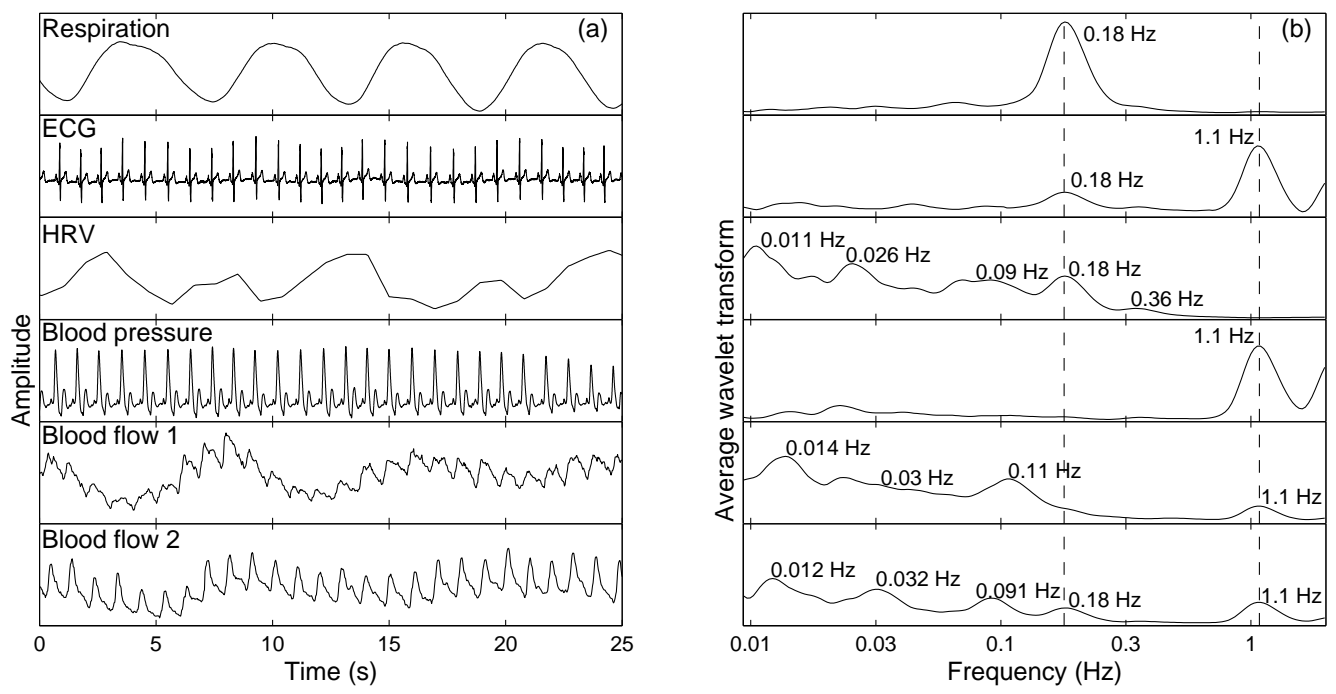

Figure 1. (a) Samples of cardiovascular signals, and (b) time averages of their wavelet transforms. The positions of the peaks are almost the same for all signals, while the corresponding amplitudes may be considerably different. After Stefanovska and Bračič. ${ }^{8}$

\subsection{Models of the cardiovascular system}

There are many earlier models of the CVS. ${ }^{1-7}$ They mostly treat it as a complex control system, and do so in terms of averaged values of blood pressure, blood flow, and other relevant quantities. They interpret the observed oscillations in the heart rate, blood pressure and blood flow in terms of nonlinearities and time delays, and concentrate mainly on short-term blood pressure control mechanisms, including respiratory oscillations and oscillations with a period of around 10s. The picture that we develop below is complementary but different.

As we will see, there is much evidence favouring the perception of the CVS as a noisy dynamical system that behaves in many respects like a collection of coupled oscillators. The phenomenological model that we will describe below in Sec. 5 takes explicit account of the dynamics of the CVS, and considers instantaneous values of the variables rather than their averages. It has the potential to capture the main dynamical features of the CVS and, it is hoped, to provide a description of the system in all its states, in sickness and in health.

In Sec. 2 we present and discuss briefly the experimental evidence for noisy oscillatory processes in the CVS. After considering their probable physiological origins in Sec. 3, we describe the interactions between the oscillators in Sec. 4. We discuss the progress made to date on modelling the CVS as a set of coupled oscillators in Sec. 5 and some applications of this approach to the CVS in Sec. 6. Finally, we draw the threads together and present our conclusions in Sec. 7 .

\section{EVIDENCE FOR OSCILLATORS}

\subsection{Cardiovascular signals}

In order to study, not just the rhythmical activity of the heart in isolation, but the CVS as a whole, it is desirable to make several different kinds of measurement simultaneously. ${ }^{8}$ Fig. 1(a) shows a short sample from a set of such results where ECG, blood pressure, and blood flow rate were recorded simultaneously, for a healthy subject in repose, over a period of 20 minutes in total. The heart rate variability (HRV) is derived from the ECG signal. 


\subsection{Spectral content - use of the wavelet transform}

Our interest centres primarily on the bloodflow circulatory control mechanisms, so processes occurring on timescales longer than about 1 minute (the average circulation period) will be ignored. Fig. 1(b) shows the averaged wavelet transform of the same data as Fig. 1(a), but calculated over the full 20 minutes. A detailed discussion of such results has been presented by Stefanovska and Bračič. ${ }^{8}$ It is immediately evident that -

- There are (at least) five characteristic spectral peaks.

- Remarkably, the same, or almost the same, peaks appear in all the spectra, regardless of where or how the corresponding signals were recorded, though there are considerable differences in amplitude.

- All the peaks are broadened.

Although the oscillatory processes implied by the spectral peaks suggest deterministic activity at those frequencies (Table 1), the widths of the peaks cannot be accounted for just in terms of the width introduced by the wavelet analysis. A clue to the origin of some of this broadening can be found by inspection of the full time-frequency wavelet spectrum, part of which is shown in Fig. 2. It can be seen that both the instantaneous amplitudes and central frequencies vary in time.

It appears that the instantaneous frequency of any given spectral peak oscillates. The modulation results mainly from interaction with the process giving rise to the spectral peak next-lowest in frequency, with contributions coming from all of the other oscillatory processes too. Heart rate variability (HRV), for example, is a signal representing the variations of the cardiac frequency, and from Fig. 1(b) we can see (third spectrum from the top) that its oscillations are also modulated by the processes with frequencies near $0.011,0.026,0.1$, and $0.18 \mathrm{~Hz}$ (as well as its own second harmonic at $0.36 \mathrm{~Hz}$ ). Such observations can be construed as evidence that the five oscillatory processes are mutually coupled. At least some of the couplings are parametric, thus giving rise to the observed frequency modulation.

The electrical activity that initiates the contraction of the heart originates at the sino-atrial node, and this periodicity has consequently been called the sinus rhythm. In adapting continuously to the needs of the body, the heart alters its rate and this alteration is known as sinus arrhythmia. HRV is extensively studied because of its potential for providing noninvasive measures of cardiovascular control mechanisms. ${ }^{10}$ However, neither the physiological mechanisms of interaction, nor their dynamical properties, are fully understood.
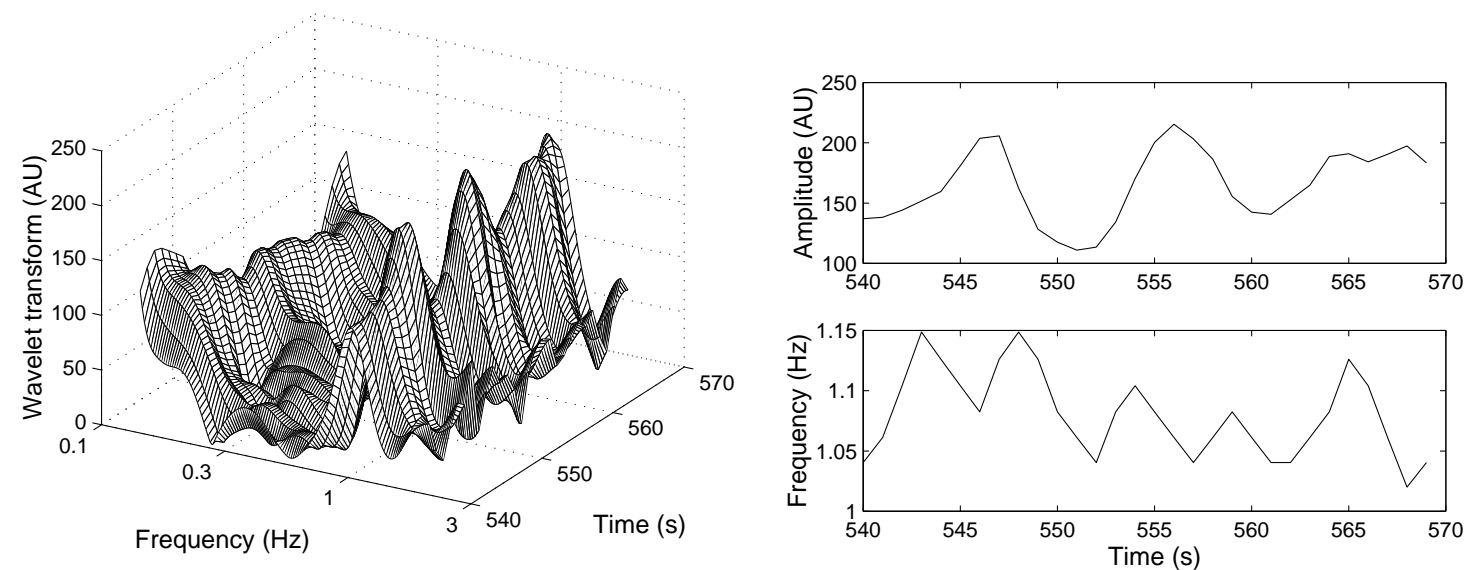

Figure 2. A segment of the wavelet transform of the blood flow signal in the time-frequency plane (left). Peaks at the heart $(\sim 1.1 \mathrm{~Hz})$ and respiration rates $(\sim 0.18 \mathrm{~Hz})$ are visible. On the right are plotted the amplitude and frequency variations of the heartrate peak. After Bračič et al. ${ }^{9}$ 


\section{PHYSIOLOGICAL ORIGINS OF THE OSCILLATIONS}

It may be inferred that the five spectral peaks probably arise from five underlying oscillatory processes of some kind. Their current physiological attributions are shown in Table 1. We consider below the reasons for

Table 1. Characteristic frequencies and their probable physiological origins.

\begin{tabular}{ll}
\hline$\sim$ Frequency $(\mathrm{Hz})$ & Physiological process \\
\hline 1.0 & Heart \\
0.2 & Respiration \\
0.1 & Intrinsic myogenic activity \\
0.03 & Neurogenic, resulting from sympathetic nerve activity \\
0.01 & Endothelial-related metabolic activity \\
\hline
\end{tabular}

these attributions, taking in turn the different spectral ranges. Before doing so, we enter three caveats. First, information about the slower three processes is obtained at one remove: they cannot be measured directly but, rather, they show up in measurements of other signals, e.g. of the ECG or blood flow. They apparently relate to mechanisms that control vasoconstriction or vasodilation (i.e. adjustments to the radii of the blood vessels), and thus are distributed throughout the whole body. Secondly, it is always possible that two or more physiological processes may be involved in maintaining a basic oscillatory activity expressed within one frequency interval, or at separate frequencies that are too close to be resolved. Thirdly, because of mutual coupling, the effect of one physiological process may manifest in several frequency intervals. Thus there must, in principle, inevitably be a measure of ambiguity in any attributions that are made.

\subsection{The $1 \mathrm{~Hz}$ and $0.2 \mathrm{~Hz}$ oscillators}

The origins of the two higher frequencies are in a sense obvious: they relate to the cardiac and respiratory processes, oscillations that can be measured directly. It is worth commenting, however, that certainty about the origin of the $\sim 0.2 \mathrm{~Hz}$ oscillations that appear in blood pressure and blood flow signals was achieved through simultaneous measurements of all these quantities, ${ }^{8}$ facilitating direct comparison of the respiratory oscillations with the apparently comparable spectral peaks in the other signals.

\subsection{The $0.1 \mathrm{~Hz}$ oscillator}

The existence of oscillations of period $\sim 10 \mathrm{~s}$ in blood pressure and HRV signals was originally attributed to blood pressure regulatory mechanisms. ${ }^{1,11}$ Reaching an understanding of the physiological origin of these oscillations is characterised, however, by two particular difficulties.

First, the $\sim 0.1 \mathrm{~Hz}$ oscillations are observed in three different contexts: as a modulation of the heart rate in the HRV signal; as waves in the blood pressure signal, long known as Mayer waves; ${ }^{12}$ and as one of the oscillatory components in blood flow through small vessels, measured by the laser Doppler technique. Conventionally, these three signals are studied separately, in the course of research in different areas of medicine such as cardiology, hypertension, or angiology, and are interpreted differently. Research on heart rate variability usually concentrates on a possible baroreceptor origin of the $0.1 \mathrm{~Hz}$ oscillatory component. Baroreceptors are biological sensors, situated in large arteries, that sense changes in pressure. Thus the $0.1 \mathrm{~Hz}$ oscillation is often believed to originate as a reflex-induced response: the interval between heartbeats increases as the arterial blood pressure rises. However, respiration also induces blood pressure fluctuations via an influence that is mainly mechanical. So it remains unclear whether baroreceptor involvement should be manifested at $\sim 0.1 \mathrm{~Hz}$ or at $\sim 0.25 \mathrm{~Hz}$ (see Frederiks et al ${ }^{?}$ and references therein) or, as we shall see shortly, at $\sim 0.03 \mathrm{~Hz}$. On the other hand, most studies of the $\sim 0.1 \mathrm{~Hz}$ oscillation, based on measurement of blood flow in small vessels, point to their local origin: they are shown to result from the intrinsic myogenic activity of smooth muscle in the walls of blood vessels, leading to vasomotion, ${ }^{13-15}$ a process that occurs even for isolated vessels in vitro. If HRV, blood pressure and blood flow 
signals and their spectra are displayed simultaneously, as presented in Fig. 1, we see that the $0.1 \mathrm{~Hz}$ oscillation can be observed in all spectra. The small differences result from their local origin.

Secondly, there is still a measure of confusion stemming from the limited frequency resolution of some of the pioneering experiments. Given the huge dynamic range of the characteristic frequencies of interest (Fig. $1(\mathrm{~b})$ ), and the fact that the frequencies also vary in time (Fig. 2), use of a conventional Fourier transform cannot separate the three lower frequencies. Consequently, all the low frequency oscillations tended to be lumped together and described as $0.1 \mathrm{~Hz}$ oscillations. In the literature, therefore, several physiological mechanisms that in reality probably manifest in lower frequency intervals, seem to compete with each other as the probable origin of the $0.1 \mathrm{~Hz}$ oscillation. Use of the recently introduced time-frequency methods with logarithmic frequency resolution $^{8}$ have brought clarity and order, however, and the new understanding is gradually replacing the conventional picture based mainly on the Fourier transform.

\subsection{The $0.03 \mathrm{~Hz}$ oscillator}

This oscillation appears to derive from neurogenic (sympathetic nervous activity, or SNA) control of the radii of the vessels. ${ }^{16}$ The best evidence comes from experiments on denervated skin flaps: it was found that, when the nerves were disconnected, the $\sim 0.03 \mathrm{~Hz}$ spectral peak disappeared. ${ }^{17} \mathrm{New}$ experimental approaches are revealing a possible role for arterial baroreceptors in the chronic regulation of SNA. ${ }^{18}$

\subsection{The $0.01 \mathrm{~Hz}$ oscillator}

There is convincing evidence ${ }^{15,19,20}$ that the $\sim 0.01 \mathrm{~Hz}$ oscillation is associated with the endothelium, the thin layer of cells that lines the inner surfaces of the blood vessels. The argument derives from laser-Doppler blood flow measurements made while diffusing different vasodilating drugs through the skin by iontophoresis. The relative power in the $\sim 0.01 \mathrm{~Hz}$ spectral peak is substantially increased when acetylcholine (ACh, an endotheliumdependent vasodilator) is administered in this way, but it is not selectively affected when the vasodilator sodium nitroprusside (SNP, a direct NO donor) is used.

\section{INTERACTIONS BETWEEN THE OSCILLATORS}

\subsection{Modulation}

Interactions among the oscillatory processes are marked by both amplitude modulation and, in particular, frequency modulation. The clearest evidence for frequency modulation can be obtained for the cardiac frequency. The HRV signal is effectively the instantaneous frequency of the heart beat and its spectrum (Fig. 1(b)) contains four characteristic frequencies, i.e. all the other four frequencies discussed above. The observed variations in the rate and the amplitude of the heart beat (as shown in Fig. 2) can thus be attributed to a continuous interaction between the oscillatory processes involved in blood distribution. ${ }^{8}$

The existence of an interaction between the cardiac and respiratory functions has been known since the 18th century when Hales carried out his celebrated experiments ${ }^{21}$ on a horse. He found that the heart rate increased on inspiration and decreased on expiration. This frequency modulation phenomenon, known as respiratory sinus arrhythmia, has been studied extensively, e.g. ${ }^{22-24}$ since then. It has also been recognized that the respiratory function can modify the amplitude of cardiac oscillations (stroke volume). ${ }^{25}$ Traube in 1865 and Hering in 1869 independently observed waves in systemic blood pressure associated ${ }^{12}$ with respiration. Their presence in both blood pressure and flow signals ${ }^{20}$ illustrates the propagation of the oscillations through the CVS. ${ }^{26}$

Although recordings can now be made noninvasively, they are in practice of insufficient duration - i.e. they do not include a large enough number of cycles - for good characterisation of the interactions between the lower frequency oscillations. 


\subsection{Cardio-respiratory synchronization}

Because the respiratory process and the heart beat may be regarded as oscillators, we may expect their mutual interaction to result in an adjustment of their rhythms, sometimes leading to synchronization. Their instantaneous frequencies can easily be measured, enabling their mutual interaction to be analysed. Early studies (see Raschke et $\mathrm{al}^{27}$ and references therein) considered temporal coordination between cardiac and respiratory rates: joint interval histograms were plotted and integer ratios of frequency and distinct phase preference were analysed. An $n: 1$ synchronization of the rhythms was found in anæsthetized rabbits, and in humans in various sleep stages, at rest, during physical work, ${ }^{27-29}$ and in anæsthesia. ${ }^{?, 30}$ It was also concluded ${ }^{31}$ that the rhythms are generally not phase-locked, implying either that the cardiorespiratory coupling is relatively weak, or that the disruptive effects of random fluctuations (noise) are relatively strong.

The introduction of nonlinear methods, and the concepts of generalised and phase synchronization, further illuminated the problem and confirmed that cardiorespiratory synchronization usually occurs as brief episodes. ${ }^{33}$ Use of the synchrogram and synchronization index facilitated the detection of synchronization even where both frequencies were time-varying. It was found that the synchronization episodes in healthy subjects at rest (e.g. Fig. 3, left) were $\sim 10$ times longer $(\sim 1000 \mathrm{~s})$ in athletes $^{33}$ than in non-athletes ${ }^{32}$ and, furthermore, that their length depended inversely on the average depth of the frequency modulation of the heart rate. From the latter result it may reasonably be inferred that the inter-oscillator coupling strength, as revealed by the lengths of the synchronization episodes, constitutes a useful piece of information about the state of the organism. This idea is apparently confirmed by measurements on a critically ill patient in coma. As shown in the right-hand panels of Fig. 3, there is absolutely no sign of synchronization: the synchrogram indicates continuous phase-slippage, even though the ratio of the cardiac and respiratory frequencies remains almost constant.

\subsection{Synchronization phenomena in anaæsthesia}

A physiological state where synchronization phenomena are of particular interest and importance is that of anaæsthesia. ${ }^{34}$ The synchronization state in a rat is then typically as shown in the measurements of Fig. 4 (left).
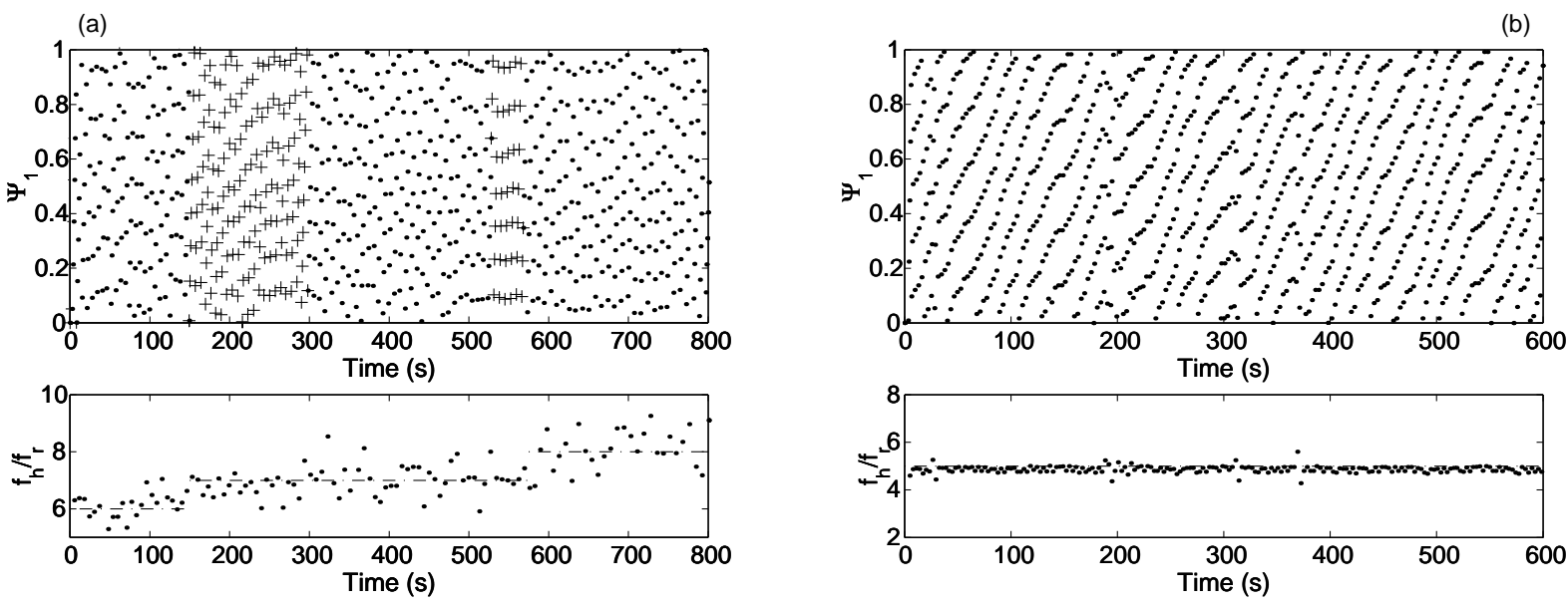

Figure 3. Comparison of cardiorespiratory synchrograms for a healthy subject and a critically ill patient in coma. (a) Healthy subject, showing the relative phase of heartbeats within respiratory cycles $\Psi_{1}$ and the instantaneous frequency ratio $f_{h} / f_{r}$. Between $510 \mathrm{~s}$ and $580 \mathrm{~s}$ seven horizontal lines are observed, indicating a 7:1 synchronization between the cardiac and respiratory oscillations. During this time, the generalised phase difference $\left|n \Phi_{h}-m \Phi_{r}\right|$, where $\Phi_{h}$ and $\Phi_{r}$ are the phases of heart and respiratory activities, remains constant and so also does the instantaneous frequency ratio. (b) The same quantities for a patient in coma. The patient was heavily sedated and ventilated via a respirator at a constant frequency of $f_{r}=0.33 \mathrm{~Hz}$. His cardiac frequency is high and constant $\left(f_{h}=1.6 \mathrm{~Hz}\right)$, resulting in a constant frequency ratio, $f_{h} / f_{r}$. However, the normalised relative phase of the heartbeat within a respiratory cycle is not constant at any time of the recording. In this critical state the systems seem to be no longer capable of responding to external perturbations, including their mutual influences, so that their phases change without any relationship one to the other. After Bračič Lotrič et al. ${ }^{32}$ 
Data recording can be initiated as soon as the anæsthetic has taken effect, at time $=0$ on the left hand side. Initially there is 1:2 synchronization, as shown by the synchrogram and by the relevant synchronization index $\lambda_{1,2}$ being close to unity. As time evolves, the anaæsthesia deepens, and the system passes through a hierarchy of different synchronization states. During deepest anaæsthesia (20-40 minutes), the system remains locked into 1:5 synchronization. This process is usually reversible, ${ }^{34}$ passing back through the same set of synchronization states as the anæsthetic wears off.
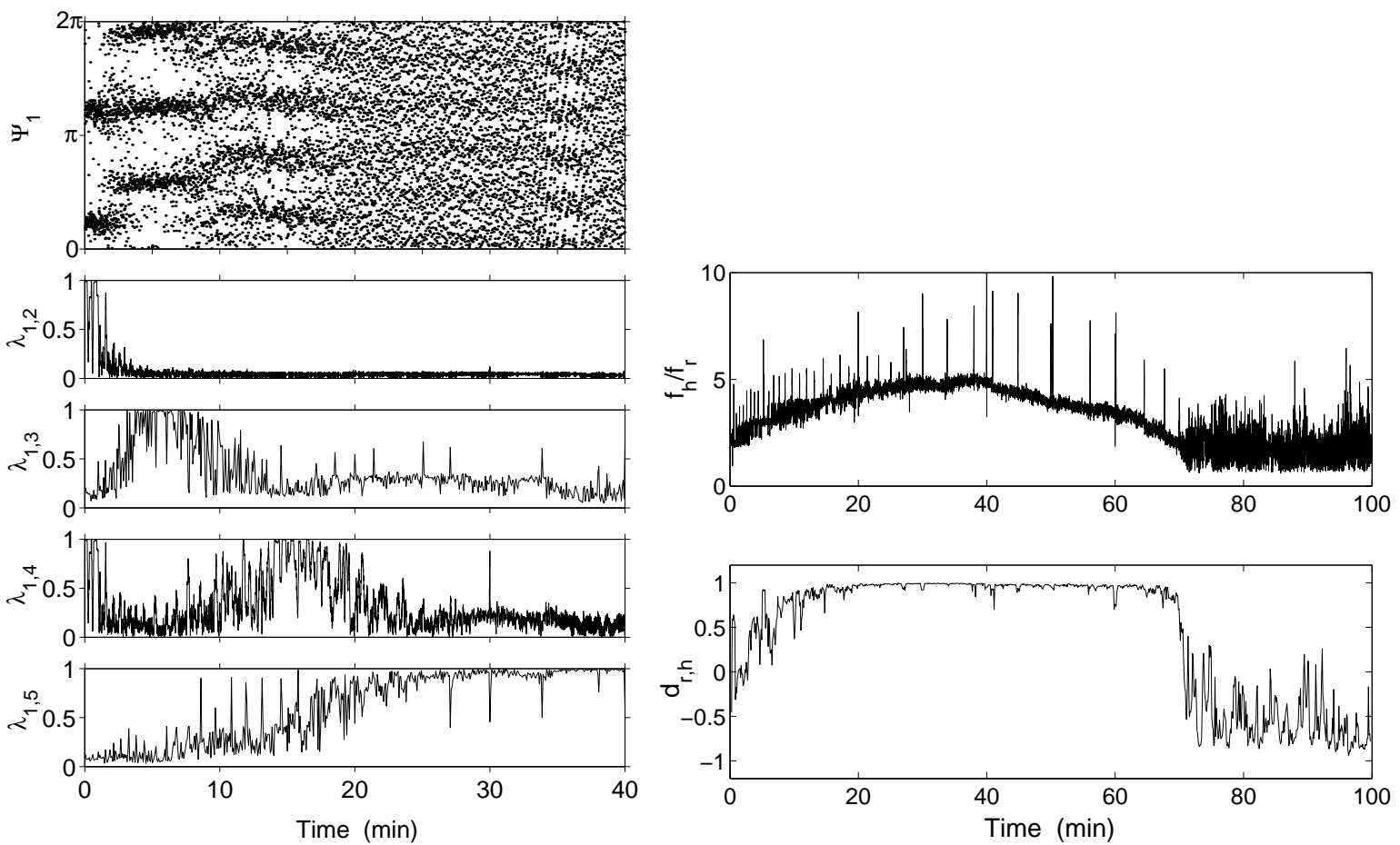

Figure 4. Left: Synchronization phenomena in an anæsthetized rat. The anæsthetic is administered at time $=-3$ min, on the left hand side. The upper panel presents the cardiorespiratory synchrogram, showing the occurrence of transitions between 1:2, 1:3, 1:4 and 1:5 synchronization states as time evolves. The anæsthesia is at its deepest at $\sim 40$ minutes. The lower four panels show the synchronization indices $\lambda_{1, n}$; perfect $1: n$ synchronization is indicated by $\lambda_{1, n}=1$. Right: Changes in the cardiorespiratory interaction in an anæsthetised rat. Top: the ratio of the cardiac $f_{h}$ and respiratory $f_{r}$ frequencies. Bottom: the directionality index $d_{r, h}$. When $d_{r, h}>0$, the direction of the interaction is such that respiration drives the heart; when $d_{r, h}<0$, the heart is driving the respiratory process, as evidently occurs at the end of deep anæsthesia.

An additional very interesting effect occurs in the cardiorespiratory interaction of rats during anæsthesia, as revealed by changes in the directionality index. ${ }^{35-38}$ This quantity relates to the causal relationships in the interaction, i.e. it enables us to distinguish between the driving and driven oscillators. The directionality index of the cardiorespiratory coupling $d_{r, h}$, calculated from the data, is plotted in Fig. 4 (right). As the anæsthetic takes effect, $d_{r, h}$ quickly rises towards unity, and remains there until consciousness starts to return again. Thus, notwithstanding the changes in the cardiac/respiratory frequency ratio $f_{h} / f_{r}$ (top), and the sequence of synchronization states being traversed, we may conclude that during deep anæsthesia respiration drives the heart, as also occurs during the waking state but that, in the shallower anæsthesia that starts after $\sim 70$ minutes, respiration is driven by the heart. This observation is not yet understood but if the same phenomenon occurs in humans, as seems likely, then there is obvious potential in using the directionality index as a noninvasive measure of depth of anæsthesia. Full details will be published elsewhere.

\subsection{Synchronization involving the lower frequency oscillators}

Given the interesting information flowing from studies of cardiorespiratory synchronization, it is natural to consider the possibility of similar phenomena between other pairs of the five oscillators. This is a challenging 
question, for several reasons. One is the practical upper limit of $\sim 30$ minutes on the length of time series that can be recorded under stationary conditions, i.e. such that the subject remains sufficiently relaxed for the cardiovascular system not to undergo large transients corresponding to movement artifacts during recording. For the lowest frequency oscillator $(\sim 0.01 \mathrm{~Hz})$ this interval includes too few complete periods to obtain good statistics in measuring a spectral density, or even to answer unambiguously whether or not synchronization exists.

There is also another quite separate problem. For the heart and respiration, separate signals are available, and these can be compared and tested for synchronization of their oscillations using the standard methods mentioned above for treating bivariate data. There is no noninvasive method for acquiring a separate signal from any of the other three oscillators, however, so that it is necessary to seek synchronization information in univariate data (a single signal). Methods proposed for doing so include: (i) decomposition into principle modes or bandpass filtration of the univariate data to synthesize two, or more "signals" 26,39 that can then be analyzed using standard methods; ${ }^{40}$ (ii) the use of angles maps; ${ }^{41}$ and (iii) bispectral analysis. ${ }^{42}$ All these approaches seem to indicate that the myogenic $(\sim 0.1 \mathrm{~Hz})$ oscillations can sometimes become synchronized with respiration.

Although no observation of synchronization involving the neurogenic or endothelial oscillations has been reported, we comment that these oscillations must be of a kind that are capable of becoming synchronized. The very fact that they can be observed ${ }^{8}$ in centrally measured quantities such as the heart rate variability signal (derived from the ECG, or the blood pressure) implies that there must be episodes, at least, of global synchronization between many of the spatially distributed oscillators of a particular frequency. Otherwise, if their phases were totally incoherent, the oscillations would average out and no spectral peak would be detected.

\subsection{Bispectral analysis}

A recent extension of bispectral analysis to encompass time dependence ${ }^{42}$ offers a very promising technique for obtaining information about the inter-oscillator interactions. It has been demonstrated by application to an interacting system of Poincaré oscillators (see Sec. 5) in the presence of noise, under a wide variety of conditions. It enables: (i) the whole frequency domain to be observed/analysed simultaneously; (ii) detection that two or more subsystems are interacting with each other; (iii) quantification of the strength of the interaction; and (iv) determination of whether the coupling is additive linear or quadratic, or parametric in one of the frequencies. Applied to the CVS, the technique has apparently demonstrated? that the cardiorespiratory interaction is nonlinear in character.

\section{COUPLED OSCILLATOR MODEL}

We now discuss in more detail the phenomenological approach to modelling the CVS introduced by Stefanovska. ${ }^{43}$ It is based on the assumption that a physiological entity that can be viewed as a "system" requires a balance between energy inflow and energy expenditure. Constant changes occur in the balance condition, resulting in oscillations. The balance may be between excitation and inhibition, as in excitable systems such as cardiac muscle, or between diffusion and reaction as is probably the case for the endothelial-based metabolic oscillations at $0.01 \mathrm{~Hz}$. She suggested that five oscillators of different frequencies, chosen to match the characteristic frequencies observed in the experiments, should be used, and the idea was elaborated in subsequent publications. ${ }^{8,8,44,44,45}$ The basic unit in the model, corresponding to the autonomous part of an oscillator, is the Poincaré oscillator

$$
\begin{aligned}
& \dot{x}_{i}=-x_{i} q_{i}-\omega_{i} y_{i}+g_{x_{i}}(\mathbf{x}), \\
& \dot{y}_{i}=-y_{i} q_{i}+\omega_{i} x_{i}+g_{y_{i}}(\mathbf{y}), \quad q_{i}=\alpha_{i}\left(\sqrt{x_{i}^{2}+y_{i}^{2}}-a_{i}\right),
\end{aligned}
$$

where $\mathbf{x}, \mathbf{y}$ are vectors of oscillator state variables, $\alpha_{i}, a_{i}$, and $\omega_{i}$ are constants and $g_{y i}(\mathbf{y})$ and $g_{x i}(\mathbf{x})$ are linear coupling vectors. Although to some extent this choice is arbitrary, (1) possesses the properties of structural stability, robustness and symmetry that are consistent with physiological understanding, and with the analyses of measured time series; it also has the advantage that the interpretation of amplitude and phase is particularly simple and direct.

The activity of each subsystem is described by two state variables, the blood flow $x_{i}$, and the velocity of flow $y_{i}$, where $i=1$ is generated by the heart, $i=2$ by respiration, $i=3$ by the myogenic oscillator, $i=4$ by the neurogenic oscillator, and $i=5$ by the endothelial related metabolic oscillator. The mutual impact of the 
subsystems is taken into account as coupling terms. Additional effects resulting from the spatial distribution of some of the systems (the myogenic, neurogenic and endothelial related metabolic activities), the effect of their propagation delays, and the influence of additional subsystems acting on longer time scales than those considered, are all taken into account as random noise.

A very important, but difficult, part of the model synthesis lies in the treatment of the couplings between the oscillators. Because the types of coupling are in most cases currently unknown, linear couplings are used, consistent with the facts that the same basic frequency components appear in all the CVS signals and that the combinatorial components appearing in them are very small. Time delays are very important in the description and modelling of the CVS. ${ }^{1-5,7,11,46,47}$ In our approach, however, we perceive the blood distribution system as one in which continuous information about every subsystem, acting with essentially different frequencies, is provided and fed back via the coupling terms. There are thus inherent delays corresponding to the time scales on which particular subsystems act. In some cases the sign of the coupling term can be determined from existing physiological knowledge or experimental observation. Direct modulation of the heart frequency by the respiration, and clear evidence of the corresponding combinational frequencies in the Fourier spectra for the subject in coma, suggest unidirectional parametric coupling, i.e. cardiac frequency modified by respiration but not vice versa. Experimental evidence also suggests that most of the couplings are asymmetrical.

The function of the heart can be represented as

$$
\begin{aligned}
& \dot{x}_{1}=-x_{1} q_{1}-y_{1} \omega_{1}+\eta_{2} x_{2}-\eta_{3} x_{3}-\eta_{4} x_{4}+\eta_{5} x_{5}-\eta_{6}\left(\Phi_{1}-\Phi_{2}\right) \\
& \dot{y}_{1}=-y_{1} q_{1}+x_{1} \omega_{1}+\eta_{2} y_{2}-\eta_{3} y_{3}-\eta_{4} y_{4}+\eta_{5} y_{5},
\end{aligned}
$$

where $\eta_{i}$ are coupling terms. Respiratory sinus arrhythmia refers to frequency modulation of the heart rate: the heart beats faster during inspiration and slower during expiration. Negative influences of the myogenic $\left(-\eta_{3}\right)$ and neurogenic system on the heart $\left(-\eta_{4}\right)$ are considered. The autonomous nervous system processes the information sensed by receptors and continuously adjusts the heart rate. The heart is innervated by both divisions of the autonomous nervous system - the sympathetic increases, and the parasympathetic decreases, the frequency and amplitude of myocardial contractions. Parasympathetic tone predominates in healthy resting humans. Increased metabolic activity results in an increased heart rate: a positive control loop is therefore assigned to the metabolic system $\left(\eta_{5}\right)$. The difference between the inflow $\left(\Phi_{1}\right)$ on the arterial side and the outflow $\left(\Phi_{2}\right)$ to the venous side also contributes to the flow of blood generated by the heart.

The dynamics of each of the other oscillators and their couplings at any point of the cardiovascular system can be described in a similar way. However, the cardiac contribution to the flow at each point results not only from the influences of local regulatory mechanisms, but also from the integration of the pressure $(P)$ and flow $(\Phi)$ values along the entire system. A global effect of the pressure on the myogenic and the neurogenic activity, and of the flow on metabolic activity, is also to be expected. Stefanovska et $\mathrm{l}^{44,45}$ therefore extended the model to describe the activity of the oscillators that regulate the flow of blood along the cardiovascular system.

\subsection{Digital modelling of the CVS}

The model equations were simulated digitally, yielding promising results ${ }^{48}$ that clearly demonstrated, however, the difficulties of optimizing parameter selection within such a large parameter space. Rather than modelling the full set of equations, we consider as an example the simplified case where we add a fluctuational term $\xi(t)$ to the cardiac oscillations (2)

$$
\begin{gathered}
\dot{x}_{1}=-x_{1} q_{1}-2 \pi f_{1} y_{1}+g_{x_{1}}(\mathbf{x})+\xi(t), \\
\langle\xi(t)\rangle=0, \quad\langle\xi(t) \xi(0)\rangle=D \delta(t),
\end{gathered}
$$

to take account of all influences coming from the rest of the system. The addition of the random term, very interestingly, resulted in occasional episodes of synchronization in the cardio-respiratory synchrogram as shown in Fig. 5, quite similar to what has been seen experimentally (cf. Fig. 3(a)). Without noise, only the standard regimes of phase-locking, phase modulation, and their interplay, could be observed. However, the modelled heart rate variability remained very small suggesting that parametric coupling probably plays an important role in the cardiac activity and in the activity of other cardiovascular oscillators. So the system was also modelled using a parametric coupling between the cardiac and respiratory oscillators. The result was regular, almost 

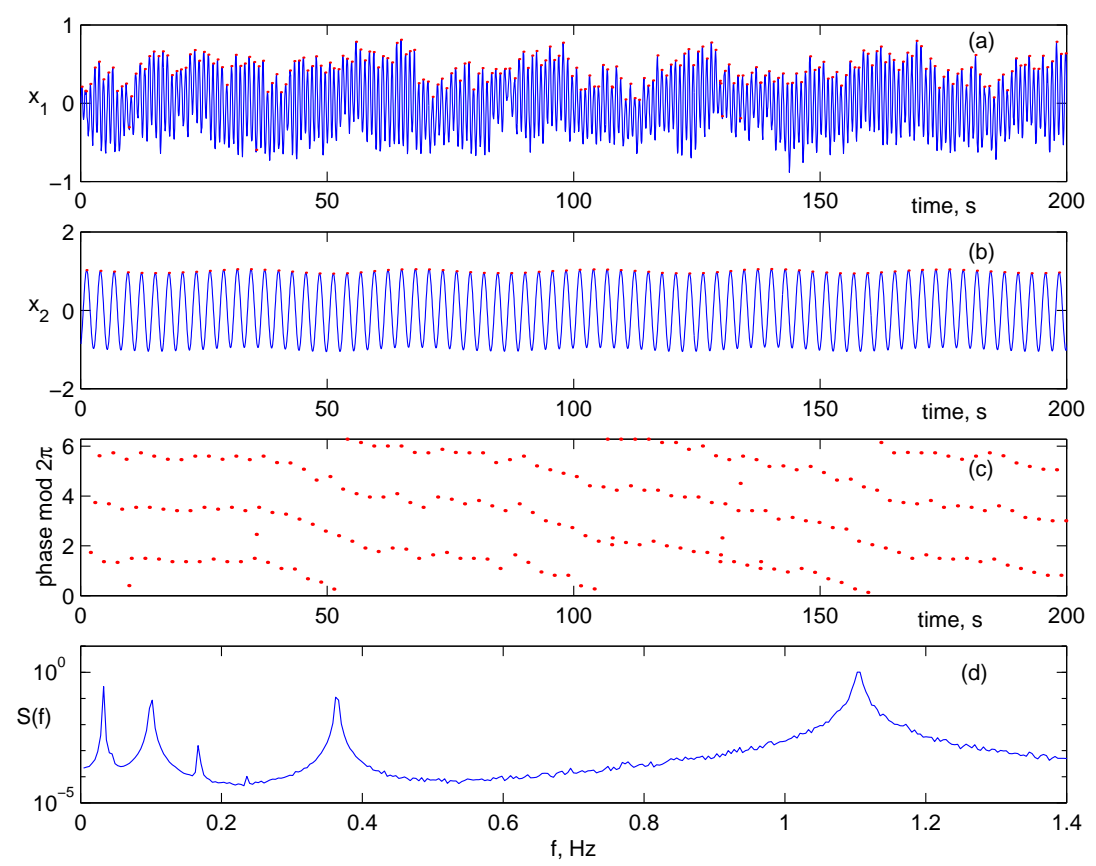

Figure 5. Results of modelling with linear couplings, in the presence of fluctuations. (a) and (b) The time series showing the rhythmic activities of the cardiac and respiratory flow components. (c) The corresponding cardio-respiratory synchrogram. (d) Power spectrum of oscillations in the blood flow generated by the cardiac activity.

periodic, episodes of synchronisation that looked quite different from the occasional episodic synchronisation seen in reality. It seems that linear couplings with noise produce a more realistic synchronisation result, but that parametric coupling is necessary to provide the depth of modulation observed in the spectra (see Fig. 2). Probably, therefore, both forms of coupling are present in reality.

\subsection{Bayesian inference}

Searching the model parameter space manually, making visual comparisons between the model data and the measurements, is time consuming and imprecise. A more systematic and objective optimisation procedure is required. One possible approach currently being explored relies on an application of Bayesian inference. The principles of this approach have already been reported ${ }^{49,50}$ in detail but, in outline, the basis of the method can be understood as follows. Consider a simple fluctuating system

$$
\begin{aligned}
& \dot{x}=K(x \mid c)+\xi(t), \\
& K(x \mid c)=\sum_{k=1}^{M} c_{k} f_{k}(x) \\
& \langle\xi(t)\rangle=0 \\
& \langle\xi(t) \xi(0)\rangle=D \delta(t)
\end{aligned}
$$

where $K(x \mid c)$ is a deterministic drift force, the $f_{k}(x)$ are known base functions with unknown coefficients $c=\left\{c_{k}\right\}$, and $\xi(t)$ is zero-mean white noise of intensity $D$. A time series obtained from it might look e.g. as shown in Fig. 6. We note immediately, first, that the time series contains a huge amount of information about the system that generated it. This example is evidently bistable; if it is a potential system, details of the shapes, depth, and coordinate separation of the two wells are contained in the fluctuations about them; details of the potential maximum separating the wells are inherent in the trajectories of the large occasional fluctuations that move the system between the wells. Secondly, this information is revealed by the noise. In the absence of noise, no information would emerge and no conclusions could be drawn. 


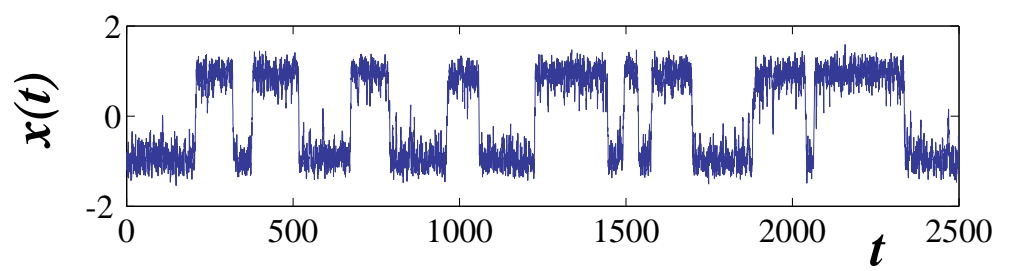

Figure 6. Example of time series from a fluctuating nonlinear system, suitable for the identification by the fast Bayesian inference technique.

The fast Bayesian inference technique ${ }^{49,50}$ proposed by Vadim Smelyanskiy formalises the approach and yields explicit values of the unknown coefficients and noise intensity. We assume that measurement error is negligible compared to the intrinsic dynamical noise $\xi(t)$, and we treat the state variable $x$ as directly observable. The conditional probability density function (PDF) $L(x \mid c)$ of an observation $x(t)$ for a given choice of the model parameters $c \operatorname{can}^{51-53}$ then be written as

$$
L(x \mid c)=P_{\mathrm{st}}\left(x\left(t_{0}\right) \mid c\right) \mathcal{F}_{c}[x(t)] .
$$

Here $P_{\mathrm{st}}(x \mid c)$ is a stationary PDF of the system $(4)$ and $\mathcal{F}_{c}[x(t)]$ is a probability density functional of $x(t)$. It can be expressed through the white noise path integral using the direct interrelation between the noise variable $\xi(t)$ and $x(t)$ given by (4). From a prior probability density function (PDF) $p(c)$ we compute a posterior PDF $p(c \mid x)$ using Bayes' theorem,

$$
p(c \mid x)=\frac{L(x \mid c) p(c)}{\int L(x \mid c) p(c) d c}
$$

where $L(x \mid c)$ is the likelihood of observing $x$ for the given parameters. The integral in the denominator in (9) ensures that the posterior PDF is normalized. Equation (9) is applied iteratively each time a new record of measurements is used: the prior distribution in the next iteration is just the posterior distribution from the previous iteration. For a sufficiently large number $N$ of observations in $x$ the posterior distribution $p(c \mid x)$ becomes sharply peaked about certain parameter values $c$ corresponding to the most probable model of the system for a given measurement set.

This approach has been tested and found to work well ${ }^{49,50,54}$ for a wide variety of simple systems where the answers are all known in advance, e.g. Duffing and Van der Pol oscillators, and a simple system with delay. In each case, rapid convergence occurred to the correct coefficients. A full application to the cardiovascular system has yet to be achieved, but the principle has already been demonstrated in relation to the respiratory system, modelled as a Poincaré oscillator. ${ }^{49,54}$ The main difficulty still to be overcome is the limited understanding of how the different physiological mechanisms interact with each other.

\section{POSSIBLE APPLICATIONS}

In the long term, it is hoped to develop a model that is capable of encompassing all states of the cardiovascular system, in health and disease, by suitable choice of parameters. The relatively simple, non-invasive, measurements described in Sec. 2.1, followed by automated analysis with a PC, could then be used for early diagnosis of a range of cardiovascular diseases and for evaluation of the effects of treatment. Already, even in the absence of a universal model, significant progress is being made in characterising a number of common states and disorders, some of which we now consider.

\subsection{Ageing}

The dynamical characteristics of the CVS change steadily with age (see Bračič Lotrič et al ${ }^{32}$ and references therein). The total spectral power of HRV signals, calculated from the wavelet transform in the interval from $0.0095 \mathrm{~Hz}$ to $0.6 \mathrm{~Hz}$, declines with age. ${ }^{32}$ A significant correlation was found between the age and the logarithm of the total power of the HRV. In addition, this decline is significant in each of the three low frequency intervals 
(around $0.01 \mathrm{~Hz}, 0.03 \mathrm{~Hz}$ and $0.1 \mathrm{~Hz}$ ). In terms of normalized power, a greater decrease of power in higher frequency intervals, around $0.1 \mathrm{~Hz}$ and $0.2 \mathrm{~Hz}$, was reported than in the interval around $0.03 \mathrm{~Hz}$ and $0.01 \mathrm{~Hz}$, implicating that the age impacts differently on the different mechanisms of cardiovascular control.

\subsection{Anæsthesia}

The marked changes in the inter-oscillator interactions of anæsthetised rats (see Sec. 4) suggest that, if similar phenomena occur in humans, there may be a possible basis for measuring depth of ancesthesia. Such instrumentation is much to be desired, given that there is currently no really satisfactory, universally accepted, method and that a statistically significant proportion of patients can subsequently recall some degree of awareness during surgery. Exploratory investigations of anæsthetised humans are currently in progress. The work is still at a very early stage, but it is already clear that cardio-respiratory synchronisation is virtually continuous in naturally breathing patients. It remains to be seen whether changes in the directionality indices are comparable with those in rats. It is also unknown whether similar changes occur in mechanically-ventilated, curarised, patients though there is no obvious reason why they should not.

\subsection{Cardiovascular diseases}

\subsubsection{Diabetes}

In diabetes, the total power of the HRV is decreased by a factor $>8$ compared to healthy subjects. The spectral power in each frequency interval is also significantly reduced. ${ }^{32}$ Both the vascular and cardiac regulatory mechanisms are modified in diabetes. The vascular changes, named angiopathy, seem to occur first, resulting in a reduced supply of blood that subsequently affects the nerve endings situated in a vessel's walls. A possible scenario is that the neural control of the vessels is then lost, resulting in increased radii, with correspondingly decreased impedance and increased blood flow. This process is named peripheral neuropathy. The cardiac nerves and small vessels undergo similar changes as well, a process that is called central autonomous neuropathy (central, to distinguish it from the changes that occur in peripheral vessels). At this stage the cardio-respiratory interactions also change. Recent studies are directed at establishing the timing of changes in cardiovascular regulation associated with diabetes. As the autonomous function is not symmetrical, these changes occur unevenly: the legs are affected first, followed by the dominant hand, while the non-dominant hand becomes affected at a later stage. We hope that the changes associated with each stage may provide a clear sequence of events to monitor, enabling us to anticipate and prevent complications such as foot ulceration or gangrene.

\subsubsection{Post-acute-myocardial-infarction}

The residual ischæmia following acute myocardial infarction was shown to reduce heart rate variability. The geometries of the beating heart and of its contraction are modified and the activity of the autonomous afferent fibres may be changed due to mechanical distortion of their sensory endings, altering cardiovascular regulation. Wavelet analysis of the post-infarction HRV revealed lower total power compared to healthy subjects, but a lesser reduction than in diabetics (about fourfold). ${ }^{32}$ The absolute power was decreased in three of the frequency intervals, around $0.01 \mathrm{~Hz}, 0.03 \mathrm{~Hz}$ and $0.1 \mathrm{~Hz}$. The most significant reduction in normalised power occurred in the interval around $0.1 \mathrm{~Hz}$, indicating that the mechanism responsible for this oscillatory component may be a major source of the decreased HRV.

\subsubsection{Cardiac failure}

Blood flow time series from cardiac failure patients are being measured on first diagnosis, prior to treatment, using iontophoretically delivered vasodilators, as described above in Sec. 2.1, to evaluate endothelial reactivity. Analysis by wavelet transform to quantify the energy in the spectral range near $0.01 \mathrm{~Hz}$ reveals that they have significantly impaired endothelial function. When the measurements/analyses are repeated a few months later, after treatment with beta-blockers has been initiated and the patients stabilised on the maximum tolerated dose, it is found that their endothelial response has changed in the direction of normality, i.e. towards the response level seen in age and gender-matched healthy subjects. 


\section{CONCLUSIONS}

Cardiovascular time series are extremely complex but, given the extensive information they must contain about the physiological state of the individual under study, they may be expected to repay careful analysis. The approach that we have reviewed above, treating the CVS as a stochastic dynamical system and applying techniques from nonlinear science, is yielding very promising results. It is revealing physiological information, e.g. on the couplings and direction of information flow between the oscillators, that are probably new to the physiologists and there appear to be several possible applications in medicine that merit further exploration and evaluation.

\section{Acknowledgements}

It is a pleasure to acknowledge valuable discussions with Andriy Bandrivskyy, Alan Bernjak, Maja Bračič, Peter Clarkson, Janez Jamšek, Yoshiki Kuramoto, Hebe Kvernmo, Dmitri Luchinsky, Bojan Musizza, Milan Paluš, Janko Petrovčič, Yuri Shiogai, Andrew Smith, Dušan Štajer and Vilma Urbančič-Rovan. The research was supported in part by the Engineering and Physical Sciences Research Council (UK), the Leverhulme Trust (UK), the British Council, and the Slovenian Ministry of Education, Science and Sport.

\section{REFERENCES}

1. B. W. Hyndman, R. I. Kitney, and B. M. Sayers, "Spontaneous rhythms in physiological control systems," Nature 233, pp. 339-341, 1971.

2. R. W. deBoer, J. M. Jaremaker, and J. Strackee, "Hemodynamic fluctuations and baroreflex sensitivity in humans: a beat-to-beat model," Am. J. Physiol. 253, pp. H680-H689, 1987.

3. G. Baselli, S. Cerutti, A. Malliani, and M. Pagani, "Cardiovascular variability signals: towards identification of a closed-loop model of a neural control mechanism," IEEE Trans. Biomed. Eng. 35, pp. 1033-1046, 1988.

4. J. P. Saul, R. D. Berger, P. Albrecht, S. P. Stein., M. H. Chen, and R. J. Choen, "Transfer function analysis of the circulation: unique insights into cardiovascular syatem," Am. J. Phyliol. 261, pp. H1231-1245, 1991.

5. B. J. TenVoorde, T. J. C. Faes, T. W. J. Jansen, G. J. Scheffer, and O. Rompelman, "Respiratory modulation of blood pressure and heart rate studied with computer model of baroreflex control," in Computer Analysis of Cardiovascular Segnals, M. D. R. et al, ed., IOS Press, Amsterdam, 1995.

6. H. Seidel and H. Herzel, "Bifurcations in a nonlinear model of the baroreceptor-cardiac reflex," Physica D 115, pp. 145-160, 1998.

7. S. Cavalcanti and E. Belardinelli, "Modelling cardiovascular variability using differential delay equation," IEEE Trans. Biomed. Eng. 43, pp. 982-989, 1996.

8. A. Stefanovska and M. Bračič, "Physics of the human cardiovascular system," Contemporary Physics 40, pp. 31-55, 1999.

9. M. Bračič, P. V. E. McClintock, and A. Stefanovska, "Characteristic frequencies of the human blood distribution system," in Stochastic and Chaotic Dynamics in the Lakes, D. S. Broomhead, E. A. Luchinskaya, P. V. E. McClintock, and T. Mullin, eds., pp. 146-153, American Institute of Physics, (Melville, New York), 2000.

10. A. J. Camm, M. Malik, J. T. Bigger, and et al., "Heart rate variability - Standards of measurement, physiological interpretation, and clinical use," Circulation 93, pp. 1043-1065, 1996.

11. R. I. Kitney, T. Fulton, A. H. McDonald, and D. A. Linkens J. Biomed. Eng. 7, pp. 217-224, 1985.

12. N. S. Cherniack, M. Heymann, and J. C. Chisholm, "Oscillations in blood pressure associalted with phrenic nerve activity," Nature 214, pp. 206-207, 1967.

13. P. C. Johnson, "The myogenic response," News Physiol. Sci. 6, pp. 41-42, 1991.

14. S. Bertuglia, A. Colantuoni, and M. Intaglietta, "Effects of L-NMMA and indomethacin on arteriolar vasomotion in skeletal-muscle microcirculation of conscious and anesthetized hamsters," Microcirc. Res. 48, pp. 68-84, 1994.

15. H. D. Kvernmo, A. Stefanovska, K.-A. Kirkebøen, and K. Kvernebo, "Oscillations in the human cutaneous blood perfusion signal modified by endothelium-dependent and endothelium-independent vasodilators," $M i$ crovasc. Res. 57, pp. 298-309, 1999. 
16. J. Karstrup, J. Bühlow, and N. A. Lassen, "Vasomotion in human-skin before and after local heating recorded with laser Doppler flowmetry - A method for induction of vasomotion," Int. J. Microcirc.: Clin. Exp. 8, pp. 205-215, 1989.

17. T. Söderström, A. Stefanovska, M. Veber, and H. Svenson, "Involvement of sympathetic nerve activity in skin blood flow oscillations in humans," Am. J. Physiol.: Heart. Circ. Physiol. 284, pp. H1638-H1646, 2003.

18. S. C. Malpas, "What sets the long-term level of sympathetic nerve activity: is there a role for arterial baroreceptors?," Am. J. Physiol.: Regulat. Integrat. and Comparative Physiol. 286, pp. R1-R12, 2004.

19. P. Kvandal, A. Stefanovska, M. Veber, H. D. Kvernmo, and K.-A. Kirkebøen, "Regulation of human cutaneous circulation evaluated by laser doppler flowmetry, iontophoresis, and spectral analysis: importance of nitric oxide and prostaglandins," Microvasc. Res. 65, pp. 160-171, 2003.

20. A. Stefanovska, M. Bračič, and H. D. Kvernmo, "Wavelet analysis of oscillations in the peripheral blood circulation measured by laser Doppler technique," IEEE Trans. Bio. Med. Eng. 46, pp. 1230-1239, 1999.

21. S. Hales, Statistical Essays II, Hamastatisticks, Innings Manby, London, 1773.

22. C. Ludwig, "Beiträge zur Kenntniss des Einflusses der Respirationsbewegungen auf den Blatlauf im Aortensysteme," Arch. Anat. Physiol. und Wiss. Med. 13, pp. 242-302, 1847.

23. A. Angelone and N. A. Coulter, "Respiratory sinus arrhythmia: A frequency dependent phenomenon," J. Appl. Physiol. 19(3), pp. 497-482, 1964.

24. J. A. Hirsch and B. Bishop, "Respiratory sinus arrhythmia in humans: How breathing pattern modulates heart rate," Am. J. Physiol.: Herat Circ. Physiol. 241, pp. H620-H629, 1981.

25. D. H. Cohoon, I. E. Michael, and V. Johnson, "Respiratory modification of the cardiac output," Am. J. Physiol. 133, pp. 642-650, 1942.

26. A. Stefanovska and M. Hožič, "Spatial synchronization in the human cardiovascular system," Progress of Theoretical Physics Supplement 139, pp. 270-282, 2000.

27. F. Raschke, "The respiratory system - Features of modulation and coordination," in Rhythms in Physiological Systems, H. H. and H. P. Koepchen, eds., pp. 155-164, Springer, Berlin, 1991.

28. G. Hildebrandt, "The autonomous time structure and its reactive modifications in the human organism," in Temporal Disorder in Human Oscillatory System, L. Rensing, U. an der Heiden, and M. C. Mackey, eds., pp. 160-174, Springer, Berlin, 1987.

29. F. Raschke, "Coordination in the circulatory and respiratory systems," in Temporal Disorder in Human Oscillatory System, L. Rensing, U. an der Heiden, and M. C. Mackey, eds., pp. 152-158, Springer, Berlin, 1987.

30. D. C. Galletly and P. D. Larsen, "Cardioventilatory coupling during anaesthesia," Brit. J. Ancsth. 79, pp. 35-40, 1997.

31. M. C. Mackey and L. Glass, From clocks to Chaos: The Rhythms of Life, Princeton University Press, Princeton, 1988.

32. M. Bračič Lotrič, A. Stefanovska, D. Štajer, and V. Urbančič-Rovan, "Spectral components of heart rate variability determined by wavelet analysis," Physiol. Meas. 21, pp. 441-457, 2000.

33. C. Schäfer, M. G. Rosenblum, and J. Kurths, "Heartbeat synchronised with respiration," Nature 392, pp. 239-240, 1998.

34. A. Stefanovska, H. Haken, P. V. E. McClintock, M. Hožič, F. Bajrović, and S. Ribarič, "Reversible transitions between synchronization states of the cardio-respiratory system," Phys. Rev. Lett. 85, pp. 4831-4834, 2000.

35. T. Schreiber, "Measuring information transfer," Phys. Rev. Lett. 85(2), pp. 461-464, 2000.

36. M. G. Rosenblum and A. S. Pikovsky, "Detecting direction of coupling in interacting oscillators," Phys. Rev. E. 64, p. 045202, 2001.

37. M. Paluš, V. Komárek, Z. Hrnčíř, and K. Štěrbová, "Synchronization as adjustment of information rates: Detection from bivariate time series," Phys. Rev. E 63, p. 046211, 2001.

38. M. G. Rosenblum, L. Cimponeriu, A. Bezerianos, A. Patzak, and R. Mrowka, "Identification of coupling direction: Application to cardiorespiratory interaction," Phys. Rev. E. 65, p. 041909, 2002.

39. P. Tass, M. G. Rosenblum, J. Weule, J. Kurths, A. S. Pikovsky, J. Volkmann, A. Schnitzler, and H.-J. Freund, "Detection of $n: m$ phase locking from noisy data: Application to magnetoencephalography," Phys. Rev. Lett. 81, pp. 3291-3294, 1998. 
40. A. Pikovsky, M. Rosenblum, and J. Kurths, Synchronization - A Universal Concept in Nonlinear Sciences, Cambridge University Press, Cambridge, 2001.

41. N. B. Janson, A. G. Balanov, V. S. Anishchenko, and P. V. E. McClintock, "Phase relationships between two or more interacting processes from one-dimensional time series: II. Application to heart-rate-variability data," Phys. Rev. E 65, pp. 036212/1-7, 2002.

42. J. Jamšek, A. Stefanovska, P. V. E. McClintock, and I. A. Khovanov, "Time-phase bispectral analysis," Phys. Rev. E 68, p. 016201, 2003.

43. A. Stefamovska, Title, PhD thesis, University of Ljubljana, Ljubljana.

44. A. Stefanovska, M. Bračič, S. Strle, and H. Haken, "The cardiovascular system as coupled oscillators?," Physiol. Meas. 22, pp. 535-550, 2001.

45. A. Stefanovska, S. Strle, M. Bracic, and H. Haken Nonlinear Phenom. in Complex Syst. 2, p. 72, 1999.

46. J. Starkee and N. W. (eds.), The Physics of Heart and Circulation, IoP Publishing, Bristol, 1993.

47. H. Seidel and H. Herzel, "Analysing entrainment of heartbeat and respiration with surrogates," IEEE Eng. Med. Biol. Mag. 17, pp. 54-57, 1998.

48. A. Stefanovska, D. G. Luchinsky, and P. V. E. McClintock, "Modelling couplings among the oscillators of the cardiovascular system," Physiol. Meas. 22, pp. 551-564, 2001.

49. A. Bandrivskyy, D. G. Luchinsky, P. V. E. McClintock, V. N. Smelyanskiy, A. Stefanovska, and D. A. Timucin, "Cardiovascular oscillations: in search of a nonlinear parametric model," in Fluctuations and Noise in Biological, Biophysical and Biomedical Systems, S. M. Bezrukov, H. Frauenfelder, and F. Moss, eds., pp. 271-281, SPIE, Washington, 2003.

50. V. N. Smelyanskiy, D. A. Timucin, D. G. Luchinsky, A. Stefanovska, A. Bandrivskyy, and P. V. E. McClintock, "Time-varying cardiovascular oscillations," in IUTAM Symposium on Nonlinear Stochastic Dynamics, N. S. Namachchivaya and Y. K. Lin, eds., pp. 455-464, Kluwer, Amsterdam, 2003.

51. R. Meyer and N. Christensen, "Bayesian reconstruction of chaotic dynamical systems," Phys. Rev. E 62, pp. 3535-3542, 2000.

52. R. Graham, "Path integral formulation of general diffusion processes," Z. Phys. B 26, pp. 281-290, 1977.

53. P. E. McSharry and L. A. Smith, "Better nonlinear models from noisy data: Attractors with maximum likelihood," Phys. Rev. Lett. 83, pp. 4285-4288, 1999.

54. A. Bandrivskyy, Fluctuations in Non-Equilibrium Systems. Inference of Stochastic and Nonlinear Cardiovascular Dynamics, PhD thesis, Lancaster University, Lancaster, 2003. 